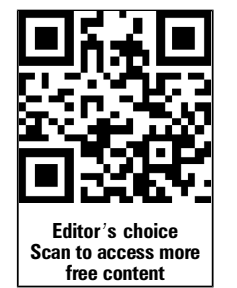

${ }^{1}$ National Poisons Information Service (Cardiff), Cardiff and Vale University Health Board, Cardiff, UK

${ }^{2}$ Institute of Molecular and Experimental Medicine, Cardiff University, Cardiff, UK

\section{Correspondence to} Dr John Paul Thompson, National Poisons Information Service Cardiff Penlan Road, Cardiff CF64 2XX, UK; ThompsonJP@cardiff.ac.uk

Accepted 15 October 2012 Published Online First 13 December 2012

\title{
Snakebite enquiries to the UK National Poisons Information Service: 2004-2010
}

\author{
James Michael Coulson, ${ }^{1,2}$ Gillian Cooper, ${ }^{1}$ Channarayapatna Krishna, ${ }^{1}$ \\ John Paul Thompson ${ }^{1,2}$
}

\begin{abstract}
Objective To describe trends regarding snakebite enquiries to the UK National Poisons Information Service (NPIS) from 2004 to 2010.

Methods The NPIS telephone enquiry database, the UK Poisons Information Database, was interrogated for enquiries to the four NPIS units from 2004 to 2010. Search terms used were 'snake' and 'snakebite'. Information from the national dataset was available from Cardiff and Edinburgh units from 2004 onwards, Birmingham from June 2005 and Newcastle from September 2006.
\end{abstract}

Results Five hundred and ten cases were identified, of which $69 \%$ were male and $31 \%$ female. Average age of cases was 32 years ( $\pm 195 \% \mathrm{Cl}$ ). The snake was identified as follows: British Adder in $52 \%$ of cases, an exotic species in $26 \%$, unknown in $18 \%$ and another UK snake in $4 \%$. $82 \%$ of cases occurred between the months of April and September. Cases peaked during August (19\%). Forty-two per cent of enquiries involved features of envenoming. Eighty-five cases were assessed as requiring antivenom. Eighty-four cases received treatment with antivenom. No adverse reactions to the antivenom were reported and resolution of clinical features was reported in all treated cases. Advice to use an antidote was followed in $98.8 \%$ of cases.

Conclusions Snakebites account for one to two NPIS cases per week. Adder bites account for over half of cases. A quarter of cases were due to non-UK snakes kept in captivity within the UK. Envenoming was said to have occurred in just under half of all cases. Advice given by the NPIS appears to closely reflect national practice guidelines.

\section{INTRODUCTION}

A recent estimate places global deaths from snakebites at a minimum of 20000 per year and acknowledges that the figure may be as high as 94000 deaths each year. ${ }^{1}$ The European adder, Vipera berus, is encountered throughout Europe and case reports and case-series of adder bites have been described from France, ${ }^{2}$ Hungary, ${ }^{3}$ Poland, ${ }^{4}$ Scandinavia, ${ }^{5}$ Turkey $^{6}$ and the UK. ${ }^{7} V$ berus is the only venomous snake that is native to the UK. The UK is therefore ideally placed to describe clinical features and the management of adder envenomation. National guidance on the management of adder bites is provided by the UK National Poisons Information Service (NPIS). ${ }^{8}$ Adherence to these recommendations at a national level has not been previously assessed in the UK.

Identification of the snake is not, however, always possible. Differentiating adders from other native species of snake is not straightforward for the layman. The possibility for encountering a snakebite injury from an exotic snake also exists. A recent trend for acquiring exotic pets has resulted in the importation of, sometimes venomous, snakes of uncertain identity into the UK. ${ }^{9}$ This may have altered the epidemiology of snakebite injuries within the UK.

$V$ berus venom contains a cocktail of hydrolytic enzymes including proteases, phospholipase, metalloproteases and phosphodiesterases. Envenoming can cause significant tissue injury, with a marked inflammatory response characterised by oedema, a risk of secondary organ injury from cardiovascular compromise and coagulopathy. ${ }^{10}$ Mortality from $V$ berus is low, approximately 14 deaths have been attributed to European adder bites since 1856, yet the morbidity can be considerable. ${ }^{7}$

Several efficacious antivenoms have been raised against $V$ berus venom. Historically there has been considerable clinical reluctance to use antivenom following the death of a child in 1957, from an anaphylactic reaction to the Pasteur Aspis-Berus antivenom administered for a mild envenoming, the conventional opinion being that 'The bite is less dangerous that the antiserum'. ${ }^{11}$

The primary objective of this study was to describe the epidemiological trends regarding snakebite enquiries and the clinical features from envenomation reported to the UK NPIS from 2004 to 2010. The secondary objective was to investigate the indications for and any complications arising from the use of Adder antivenom were also considered by comparing the clinical features of each case with the indications for treatment with respect to NPIS treatment guidelines. ${ }^{8}$

\section{METHODS}

In the UK, clinical advice and the location of stocks of antivenom is available through the NPIS. Telephone enquiries to the NPIS are electronically recorded in the UK Poisons Information Database. This database records the demographic, clinical features, any treatment given or recommended and, when possible, the outcome of each telephone enquiry. The UK Poisons Information Database was interrogated for enquiries to the four NPIS units from 2004 to 2010. Search terms used were 'snake' and 'snakebite'. Information from the national dataset was available from Cardiff and Edinburgh units from 2004 onwards, Birmingham from June 2005 and Newcastle from September 2006. Data concerning snakebites in animals or from snakebite injuries acquired from outside the UK were removed. The data were analysed using SPSS version 18.0 statistics and presented as mean 
$( \pm 95 \% \mathrm{CI})$. The $\chi^{2}$ statistic was used to compare proportions. A p-value of $<0.05$ was considered statistically significant.

For each adder bite that was treated with antivenom, the indication for treatment and any complications following treatment with the antivenom were recorded. A retrospective review of each potential adder bite identified was conducted to determine the agreement of treatment decisions with practice guidelines. The clinical features were reviewed by an investigator blinded to any subsequent treatment and a decision to treat with antivenom was made by reference to the NPIS treatment guidelines. $^{8}$

\section{RESULTS}

Five hundred and twenty-two enquires of snakebite injury in humans were identified between 2004 and 2010. These referred to 510 cases, an average of 72 cases a year ( \pm 12 cases).

The snake was identified as the British Adder in $52 \%$ of cases, an exotic species in $26 \%$ of cases, unknown in $18 \%$ and another native UK snake (Coronella austriaca or Natrix natrix) in $4 \%$ of cases. All of the injuries that resulted from exotic species were from privately owned snakes. The most frequently encountered were the corn or rat snake (Pantherophis guttatus) $(27 \%)$, Boas (20\%), Pythons (20\%) and Western hognose (Heterodon nasicus) (11\%). Three per cent of exotic snakebites were from venomous snakes: the western diamond rattlesnake (Crotalus atrox) and the eastern green mamba (Dendroaspis angusticeps).

Eighty-two per cent of cases occurred between the months of April and September, with a peak incidence occurring during August (19\% of cases). The incidence of adder bite enquiries was greatest in the summer months, $p<0.00001$. There was no difference in the monthly distribution of exotic snakebite enquires, $p=0.06$.

The average age of patient was 32 years ( \pm 1 year). The age range was from less than 12 months to 87 years of age. Twenty-six per cent of cases were children below the age of 16 years. There was a male predominance: $69 \%$ were male and $31 \%$ female, $p=0.000001$. The site of injury was identified as the upper limb (hand) in 32\% of enquires the lower limb (foot) in $14 \%$ and the head or neck in $1 \%$. In $53 \%$ of enquires, the site of injury could not be determined from the records. The male to female ratio of snakebite injuries to the hand was 3.6 to $1, p=0.008$.

Forty-two per cent of enquiries reported features of envenoming. All cases that resulted in envenoming were from adder bites. Clinical features in those with envenoming were reported as follows: $94 \%$ oedema, $28 \%$ cardiovascular signs (14\% hypotension and tachycardia, $8 \%$ hypotension and 6\% tachycardia), $23 \%$ gastrointestinal features (vomiting and diarrhoea) and $7 \%$ had anaphylaxis (defined as hypotension with one or more features of orofacial oedema, rash or bronchospasm). Thirteen per cent were reported as having a leucocytosis. Only two cases were reported to have had abnormal ECG (both had dynamic T-wave inversion). No deaths or significant coagulopathies were reported.

Eighty-five cases were assessed by clinicians, in consultation with the NPIS, as requiring treatment with antivenom. The most frequent indications for treatment with antivenom were: oedema involving two joints (64\%), hypotension (29\%) and anaphylactoid reaction (7\%). Eleven per cent of these cases also had biochemical abnormalities (neutrophilia, raised creatine kinase or metabolic acidosis).

Eighty-four cases received treatment with antivenom. In one case, the patient refused treatment with antivenom due to concerns of a possible allergy to the antivenom. Advice to use an antidote was therefore followed in $98.8 \%$ of cases. The Zagreb antivenom was administered in each case. No adverse reactions to the antivenom were reported and resolution of clinical features was reported in all, antivenom treated cases. Only two of these cases required treatment with a further two ampoules of antivenom, due to continuing envenoming.

A retrospective review of the 265 reported cases of adder bites identified 89 cases that met the NPIS guidelines for treatment with antivenom and 176 cases where treatment would have been inappropriate. The indications were: oedema involving two joints (56\%), hypotension (16\%), anaphylaxis $(6 \%)$, neutrophilia (12\%), raised creatine kinase $(6 \%)$, metabolic acidosis $(2 \%)$ and $\mathrm{T}$-wave inversion on the electrocardiogram (2\%). The difference between the 89 cases identified for treatment during the retrospective review and the 85 actual cases, where there was an intention to treat, was due to the presence of an isolated neutrophilia.

\section{DISCUSSION}

The true incidence of snakebite injuries in the UK is unknown. Several detailed case reviews exist, ${ }^{711} 12$ but these are based on the experiences of individual clinicians or institutes and focus on adder bites. A single study estimated the UK annual incidence of $V$ berus snakebite injuries at more than 90 cases from an analysis of both published and unpublished data from the regional health authorities and the NPIS. ${ }^{13}$

The majority of snakebite injuries referred to the NPIS were due to adder bites. This was not unsurprising given that the adder is the only venomous native snake. We described a higher incidence of injuries from exotic snakes than an older UK study. ${ }^{14}$ This may reflect a rise in the number of snakes kept by private individuals. The vast majority of these were either nonvenomous or secreted a venom of very low toxicity to humans. Forty-three cases of exotic snakebites were reported to Professor Warrell, the UK's leading authority on snakebite injuries, between 1986 and 2009 and this probably represents an underestimate of the true number. ${ }^{15}$

Our study included all species of snakebite injuries and was based on enquires to the NPIS. Data was only available from the Birmingham NPIS from June 2005 and from Newcastle from September 2006. This probably represents an underreporting of the true number of snakebite injuries. This may be due to a selection bias in the presentation of individual cases. A Scandinavian study suggests that only a minority of cases seek medical aid following snakebite injury. ${ }^{16}$

Previous studies of the incidence of snakebite injuries have described seasonal variations in the presentation of adder bites. ${ }^{7} 13$ This trend probably reflects the hibernation patterns of $V$ berus since it did not extend to exotic snakes in our study, contact with which was always in the home environment.

Studies of the epidemiology of UK adder bites have described a higher case frequency of snakebite injuries in males compared with females and a tendency by males to be bitten on the hand. ${ }^{71-13}$ While our data is consistent with this trend, in over half of reported cases, the site of injury could not be determined from the database entry.

Envenoming does not appear to be an inevitable consequence of a snakebite injury. The majority of cases are often asymptomatic. ${ }^{13}$ Envenoming was reported in $42 \%$ of cases in our study and was often characterised by tissue oedema and cardiovascular stress (hypotension and tachycardia). This is consistent both with the effects of the venom in experimental 
primates, ${ }^{10}$ case reports ${ }^{12} 13$ and case-series from both the $\mathrm{UK}^{7}$ and Europe. ${ }^{17} 18$

Zagreb antivenom is a $\mathrm{F}(\mathrm{ab}) 2$ antibody purified from equine serum that can prevent mortality in experimental animals exposed to four times the lethal dose of $V$ berus venom ${ }^{10}$ and reduce both morbidity and inpatient duration in humans. ${ }^{9}$ The recommended dose of Zagreb antivenom is two ampoules. ${ }^{8}$ One ampoule of Zagreb $(5.4 \mathrm{ml})$ is capable of neutralising $9 \mathrm{mg}$ of venom. ${ }^{10}$ A juvenile adder can administer between $10-18 \mathrm{mg}$ of venom with a single bite. The two cases that required a further dose of antidote may have received a larger dose of venom at the time of injury. An alternative explanation may be due to pharmacokinetic differences between the half-life of the venom and the half-life of the antivenom.

Non-IgE mediated hypersensitivity reactions may complicate about $10 \%$ of patients treated with antivenom. ${ }^{19}$ No such adverse reactions were reported in our study, although we did not exclude the possibility of coadministration of agents such as adrenaline that may have attenuated a hypersensitivity response. ${ }^{10}$ The lack of adverse reactions to the antivenom may have reflected high concordance between the decision to treat and compliance with the national guidance.

\section{CONCLUSION}

Snakebite injuries accounted for approximately one to two enquiries to the UK NPIS each week between 2004 and 2010. Adder bites accounted for over half of cases. Approximately one quarter of cases were due to non-UK snakes kept in captivity within the UK. Envenoming was said to have occurred in just under half of all cases in our study. Oedema was the most significant feature, although systemic features also occurred in over half of the cases. The antivenom appears to be safe and effective. Advice given by the NPIS appears to closely reflect national practice guidelines.

Acknowledgements We would like to thank our colleagues in the National Poisons Information Service centres at Birmingham, Edinburgh and Newcastle for allowing us access to their data. The National Poisons Information Service is commissioned by the Health Protection Agency of the United Kingdom.

Contributors JMC planned the study, analysed the data and wrote the manuscript. GC collected the data. JPT and CK were the custodians of the data. JMC and JPT take responsibility for the overall content as guarantors.
Funding None.

Competing interests None.

Provenance and peer review Not commissioned; externally peer reviewed.

Data sharing statement The National Poisons Information Service centres in Birmingham, Edinburgh and Newcastle for supplying their data.

\section{REFERENCES}

1. Kasturiratne A, Wickremasinghe AR, de Silva N, et al. The global burden of snakebite: a literature analysis and modelling based on regional estimates of envenoming and deaths. PLoS Med 2008;5:e218.

2. de Haro L. Aggravation of snake bite in France and their treatment. Presse Med 2003;32:1131-7.

3. Malina T, Krecsak L, Warrell DA. Neurotoxicity and hypertension following European adder (Vipera berus berus) bites in Hungary: case report and review. OMJ 2008;101:801-6.

4. Magdalan J, Trocha M, Merwid-Lad A, et al. Vipera berus bites in the region of southwest Poland-a clinical analysis of 26 cases. Wilderness Environ Med 2010;21:114-19

5. Grönlund J, Vuori A, Nieminen S. Adder bites. A report of 68 cases. Scand J Surg 2003;92:171-4.

6. Al B, Orak M, Aldemir M, et al. Snakebites in adults from the Diyarbakir region in southeast Turkey. Ulus Travma Acil Cerrahi Derg 2010;16:210-14.

7. Reid HA. Adder bites in Britain. BMJ 1976;2:153-6.

8. British National Formulary. British Medical Association and Royal Pharmaceutical Society of Great Britain. 2011, No. 61.

9. Warrell DA. Treatment of bites by adders and exotic venomous snakes. BMJ 2005;331:1244-7.

10. Theakston RD, Reid HA. The effectiveness of Zagreb antivenom against envenoming by the adder vipera berus. Lancet 1976;2:121-3.

11. McKiddie MT. Treatment of adder bites. BMJ 1979;6:865-6.

12. Hawley A. Adder bites in the British Army, 1979-1988. J R Army Med Corps 1990;136:114-18.

13. Reading CJ. Incidence, pathology and treatment of adder (Vipera berus L.) bites in man). J Accid Emerg Med 1996;13:346-51.

14. Reid HA. Bites by foreign venomous snakes in Britain. BMJ 1978;1:598-600.

15. Warrell DA. Commissioned article: management of exotic snakebites. OMJ 2009;102:593-601.

16. Marquart H. Recherches statistiques sur les accidents par morsures de serpents au Danemark et en Suède de 1900 à 1947. Presse Med 1951;59:1110-11.

17. Persson H, Irestedt B. A study of 136 cases of adder bites in Swedish hospitals during one year. Acta Meda Scand 1981;210:433-9.

18. Karlson-Stiber C, Salmonson H, Persson H. A nationwide study of Vipera berus bites during one year-epidemiology and morbidity of 231 cases. Clin Toxicol (Phila) 2006;44:25-30.

19. Karlson-Stiber C, Persson H. Antivenom treatment in Vipera berus Envenomingreport of 30 cases. J Intern Med 1994;235:57-61. 